\title{
Chronic Active Antibody-Mediated Rejection with Linear IgG Deposition on Glomerular Capillaries in a Kidney Transplant Recipient
}

\author{
Kenichiro Miura ${ }^{a}$ Yoko Shirai ${ }^{a} \quad$ Naoto Kaneko ${ }^{a}$ Tomoo Yabuuchi ${ }^{a}$ \\ Kiyonobu Ishizuka $^{a}$ Shigeru Horita ${ }^{b}$ Miyuki Furusawac Kohei Unagamid \\ Masayoshi Okumi $^{\mathrm{e}}$ Hideki Ishidad Kazunari Tanabe $^{\mathrm{c}}$ Junki Koike ${ }^{\mathrm{f}}$ \\ Kazuho Hondag Yutaka Yamaguchi ${ }^{\text {h }}$ Motoshi Hattori ${ }^{\text {a }}$ \\ aDepartment of Pediatric Nephrology, Tokyo Women's Medical University, Tokyo, Japan; 'Department of Pathology, \\ Kidney Center, Tokyo Women's Medical University, Tokyo, Japan; 'Department of Urology, Tokyo Women's Medical \\ University, Tokyo, Japan; 'Department of Organ Transplant Medicine, Tokyo Women's Medical University, Tokyo, \\ Japan; 'Department of Urology, Osaka Police Hospital, Osaka, Japan; 'Department of Diagnostic Pathology, St. \\ Marianna University School of Medicine, Kawasaki, Japan; 9Department of Anatomy, Showa University School of \\ Medicine, Tokyo, Japan; 'Yamaguchi's Pathology Laboratory, Chiba, Japan
}

\section{Keywords}

Antibody-mediated rejection - Endothelial cells · lgG .

Kidney transplantation - Non-HLA antibody

\begin{abstract}
Glomerular IgG deposition is rarely observed in antibodymediated rejection. Here, we report chronic active antibodymediated rejection with linear IgG deposition on glomerular capillary walls in a pediatric kidney transplant recipient. A 6-year-old boy with bilateral renal hypoplasia underwent preemptive deceased-donor kidney transplantation. Five years after the transplantation, an allograft biopsy revealed chronic active antibody-mediated rejection with diffuse linear IgG deposition on glomerular capillaries. Anti-glomerular basement membrane antibody, donor-specific anti-human leukocyte antigen (HLA) antibodies, and anti-angiotensin II type 1 receptor antibody were negative. A multiplex antibody assay identified anti-major histocompatibility complex class I chain-related molecule A antibody. Additionally, a single-antigen bead assay identified autoantibodies to
\end{abstract}

12 non-HLA antigens, including vimentin and glutathione S-transferase theta-1. To investigate whether IgG autoantibodies in the patient's serum bind to antigens on glomerular capillaries, we incubated the patient's serum with 0 -h biopsy specimens of tissue donated to the patient and a control subject, both obtained immediately after nephrectomy from respective donors. IgG signals were observed in neither patient nor control samples. Nevertheless, linear IgG deposition may be explained by the binding of autoantibodies to non-HLA antigens that are usually hidden and only exposed via severe endothelial cell injury. Further studies are needed to confirm the significance of non-HLA antibodies in glomerular IgG deposition.

(c) 2020 S. Karger AG, Basel

\section{Introduction}

Chronic active antibody-mediated rejection (CAAMR) is an important risk factor for allograft dysfunction and graft loss after kidney transplantation. In karger@karger.com www.karger.com/nef

(c) 2020 S. Karger AG, Base

Karger"
Motoshi Hattori

Department of Pediatric Nephrology, Tokyo Women's Medical University 8-1 Kawada-cho, Shinjuku-ku

Tokyo 162-8666 (Japan)

hattori@twmu.ac.jp 

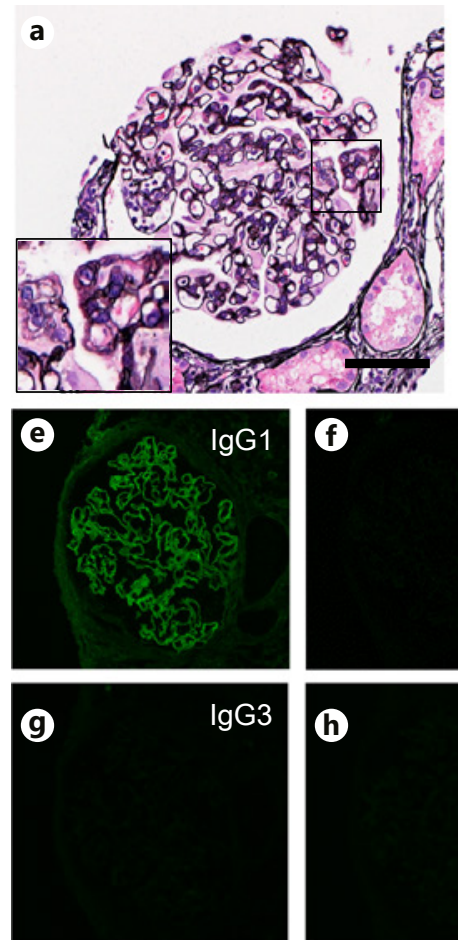
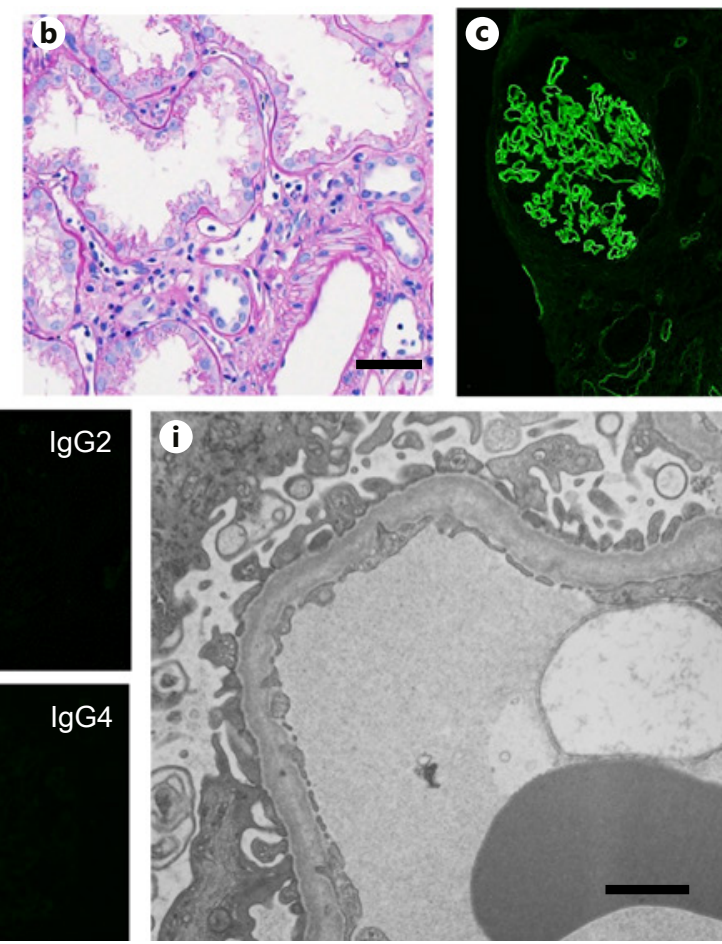
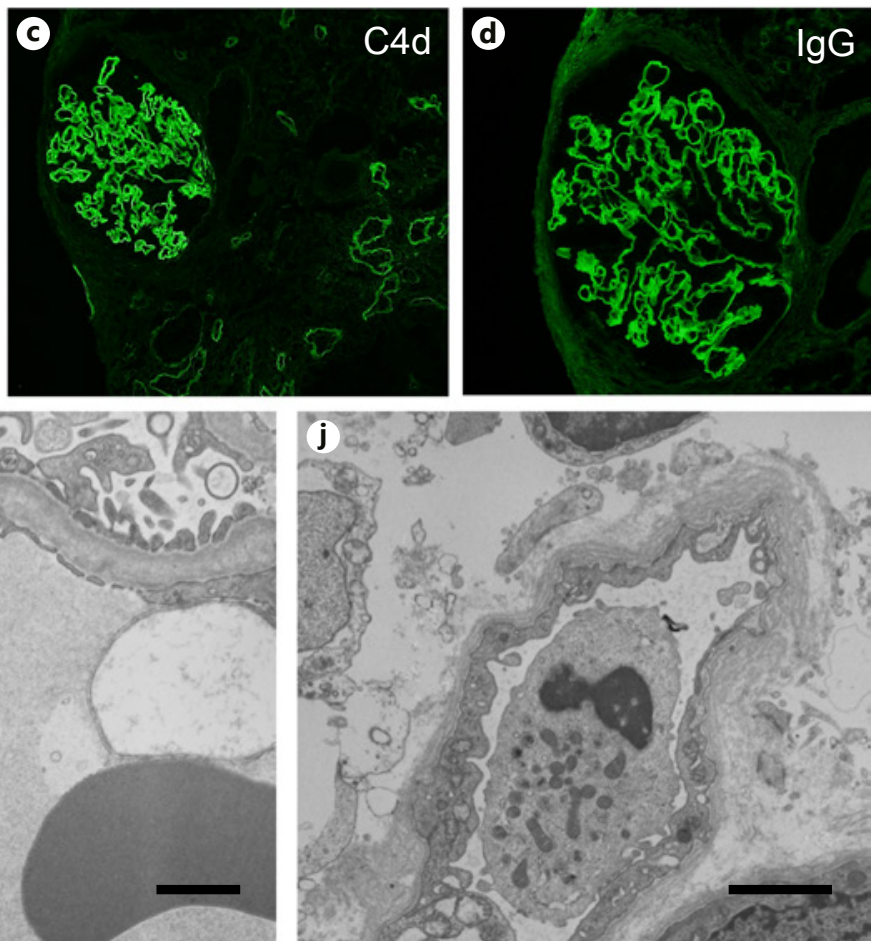

Fig. 1. Allograft biopsy 5 years post-kidney transplantation. a Light microscopy shows partial duplication of the glomerular capillary walls and mild glomerulitis (periodic acid-methenamine-silver stain, original magnification, $\times 400)$. Higher magnification of the marked area shows double contour of the capillary walls. Scale bar denotes $50 \mu \mathrm{m}$. b Moderate peritubular capillaritis and peritubular capillary basement membrane thickening (periodic acid-Schiff stain, original magnification, $\times 400$ ). Scale bar denotes $50 \mu \mathrm{m}$. c Immunofluorescence shows diffuse C4d staining along the glomeru- lar capillary walls and peritubular capillaries. d Immunofluorescence shows intense linear signals for IgG along capillary walls. e-h IgG subclass analysis showed positive IgG1 signals, while signals for IgG2, IgG3, and IgG4 were negative. i Electron microscopy shows prominent subendothelial space widening with duplication of the GBM. No electron-dense deposits were observed. Scale bar denotes $2 \mu \mathrm{m}$. $\mathbf{j}$ Electron microscopy of the peritubular capillary shows multilayering of the basement membrane. Scale bar denotes $2 \mu \mathrm{m}$. GBM, glomerular basement membrane. most patients with CAAMR, anti-human leukocyte antigen (HLA) donor-specific antibodies (DSAs) are involved. However, this type of rejection can also occur in patients who are DSA negative. Mounting evidence points to the role of non-HLA antibodies as important contributors to CAAMR [1]. The pathological findings of CAAMR include transplant glomerulopathy, peritubular capillary basement membrane multilayering, and arterial intimal fibrosis. CAAMR is also characterized by microvascular inflammation or C4d deposition on peritubular capillary walls $[1,2]$. Glomerular IgG deposition, however, is rarely observed in CAAMR [2]. Here, we report a pediatric kidney transplant recipient with nonHLA antibodies, but not anti-HLA DSAs, who developed CAAMR with linear IgG deposition on glomerular capillary walls.

\section{Case Report}

A 6-year-old boy with bilateral renal hypoplasia underwent preemptive deceased-donor kidney transplantation. His EpsteinBarr virus (EBV) serostatus was donor-positive/recipient-negative. Immunosuppressive regimens consisted of basiliximab, tacrolimus, mycophenolate mofetil, and methylprednisolone. The estimated glomerular filtration rate (eGFR) was $40-50 \mathrm{~mL} /$ $\min / 1.73 \mathrm{~m}^{2}$. Per-protocol allograft biopsies at 1 - and 6 months post-kidney transplantation showed mild interstitial fibrosis and tubular atrophy, which were likely due to ischemia. The plasma EBV-DNA load was approximately $1.0 \times 10^{4}$ copies $/ 10^{6}$ cells 6 months post-kidney transplantation, which prompted us to reduce the dosage of immunosuppressive agents [3]. The trough levels of tacrolimus and mycophenolate mofetil were $2.0-2.5 \mathrm{ng} / \mathrm{mL}$ and $1.0-3.0 \mathrm{ng} / \mathrm{mL}$, respectively.

At 3.5 years post-kidney transplantation, eGFR decreased to 39 $\mathrm{mL} / \mathrm{min} / 1.73 \mathrm{~m}^{2}$. An allograft biopsy showed CAAMR with a low level of linear IgG deposition on glomerular capillary walls. The Banff classification was i1, t0, v0, g0, ptc2, ci0, ct0, cv0, cg0, ptcbm1, ah0, ah0, and C4d0.
Miura et al. 
Fig. 2. Immunofluorescence was performed to analyze the binding of autoantibodies in patient serum to glomerular capillary walls. Sera of the patient (1:40) and a healthy control (1:40) were incubated with 0 -h allograft biopsy specimens from the patient and a control subject which were obtained immediately after nephrectomy from donors. Secondary antibody was fluorescein isothiocyanate-conjugated rabbit anti-human IgG (1:50). No significant signals for IgG were observed in both 0 -h specimens of the patient (a) and the control subject (b) that were incubated with the patient's serum. Similarly, no significant signals for IgG were observed in both 0 -h specimens of the patient (c) and the control subject (d) that were incubated with serum from a healthy control.

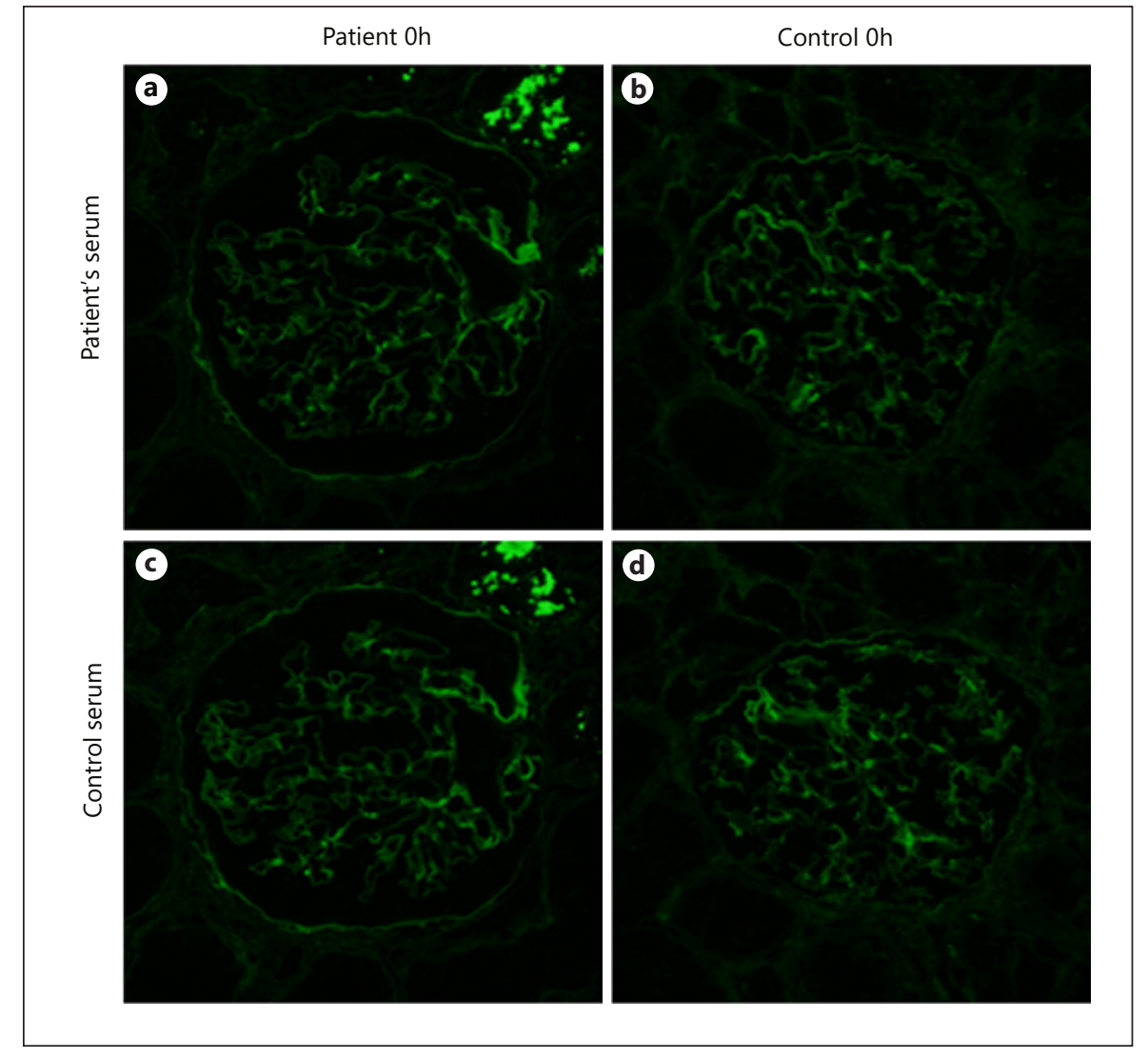

Table 1. Antigens analyzed by Luminex LABScreen Autoantibody assay

\begin{tabular}{|c|c|}
\hline Detected & Not detected \\
\hline $\begin{array}{l}\text { Alpha-enolase, C-X-C motif chemokine 9, eukaryotic translation } \\
\text { initiation factor } 2 \mathrm{~A} \text {, glial cell-derived neurotrophic factor, } \\
\text { glutathione S-transferase theta-1, heterogeneous nuclear, } \\
\text { peroxisomal trans-2-enoyl-CoA reductase, protein kinase C eta, } \\
\text { protein kinase C zeta, protein tyrosine phosphatase receptor type N, } \\
\text { regenerating islet-derived protein } 3 \text { alpha, ribonucleoprotein K, and } \\
\text { vimentin }\end{array}$ & $\begin{array}{l}\text { Agrin, angiotensinogen, aurora kinase A, C-X-C motif chemokine } 10, \mathrm{C}-\mathrm{X}-\mathrm{C} \text { motif } \\
\text { chemokine } 11 \text {, chromatin assembly factor } 1 \text { subunit B, fibronectin leucine-rich } \\
\text { transmembrane protein } 2 \text {, glyceraldehyde-3-phosphate dehydrogenase, interferon } \\
\text { gamma, interferon induced with helicase C domain } 1 \text {, lamin A/C, lamin B1, } \\
\text { myosin-binding protein C, nucleolar and spindle associated protein } 1 \text {, } \\
\text { peptidylprolyl isomerase A, perlecan, platelet glycoprotein } 5 \text {, Rho GDP- } \\
\text { dissociation inhibitor 2, secretory phospholipase A2 receptor, tubulin alpha-1B } \\
\text { chain, and tumor necrosis factor alpha }\end{array}$ \\
\hline
\end{tabular}

The presence of non-HLA antibodies was investigated using Luminex LABScreen Autoantibody (One Lambda) which can simultaneously detect up to 33 autoantibodies. Data acquisition and analysis were performed using the LABScan 3D flow analyzer. HLA, human leukocyte antigen.

Five years posttransplantation, eGFR further decreased to 31 $\mathrm{mL} / \mathrm{min} / 1.73 \mathrm{~m}^{2}$ due to non-adherence. There were no laboratory findings suggestive of thrombotic microangiopathy (e.g., thrombocytopenia, hemolytic anemia, and elevated lactate dehydrogenase levels). Serum anti-glomerular basement membrane (GBM) antibody was negative and hemoglobin A1c level was normal. An allograft biopsy showed mild glomerulitis, double contour of the glomerular capillary walls, moderate peritubular capillaritis, and peritubular capillary basement membrane thickening (Fig. 1a, b). No pathological changes suggestive of diabetic nephropathy such as polar vasculosis, mesangial matrix increase, nodular lesion, and microaneurysm were observed. There was a strong immunofluorescence signal for $\mathrm{C} 4 \mathrm{~d}$ on glomerular capillaries and peritubular capillaries (Fig. 1c). All of the above findings were compatible with CAAMR. In addition, the Banff classification was $\mathrm{i} 0, \mathrm{t} 0, \mathrm{v} 0, \mathrm{~g} 1$, ptc2, ci2, ct2, cv0, cg1, ptcbm1, mm0, ah0, aah0, ti2, i-IFTA2, and C4d3, and immunofluorescence showed diffuse linear IgG deposition on glomerular capillary walls (Fig. 1d). Immunofluorescence study performed at the same time showed that signals for IgG was negative in kidney biopsy specimens obtained from 2 patients with 
IgA nephropathy and 1 patient with secondary focal segmental glomerulosclerosis after kidney transplantation (data not shown). In the present patient, the signals for IgM, the third component of complement $(\mathrm{C} 3)$, and $\mathrm{C} 1 \mathrm{q}$ were negative. IgG subclass staining showed that IgG1 was positive, while IgG2, IgG3, and IgG4 were negative (Fig. 1e-h). No light-chain isotype restriction was observed. Electron microscopy showed diffuse subendothelial widening with partial duplication of the GBM (Fig. 1i). Peritubular capillary basement membrane multilayering was also noted (Fig. 1j). Electron-dense deposits were not identified.

Because the patient was anti-HLA DSA negative, we searched for non-HLA antibodies. A quantitative ELISA (One Lambda, West Hills, CA, USA) did not detect anti-angiotensin II type 1 receptor antibodies. Luminex microbead technology using LABScreen Mixed assay (One Lambda) detected anti-major histocompatibility complex class I chain-related molecule A (MICA) in the patient's serum. We further searched for other non-HLA antibodies using Luminex LABScreen Autoantibody (One Lambda). Twelve autoantibodies including vimentin and glutathione Stransferase theta-1 (GSTT1) were detected (Table 1).

To investigate whether IgG autoantibodies in the patient's serum bind to antigens on glomerular capillaries, we incubated patient serum and serum from a healthy control with 0 -h donortissue biopsy specimens of the patient and a control (obtained immediately after nephrectomy from respective donors). IgG signals were not observed in patient or control biopsy specimens that were incubated with patient serum (Fig. 2). The patient and his parents gave written informed consent to participate in the study.

\section{Discussion}

The patient described here developed CAAMR following kidney transplantation. This was presumed to be due to a reduction of the dosage of immunosuppressive agents. An allograft biopsy showed linear IgG deposition on glomerular capillary walls, which has not been previously described in CAAMR.

Linear IgG staining on glomerular capillary walls is a characteristic finding of anti-GBM disease. However, the patient was anti-GBM antibody negative, and allograft biopsy specimens showed no glomerular crescent formation. Linear staining of IgG, along with IgM and complement C3, is also observed in diabetic nephropathy. This is thought to reflect structural changes in endothelial cells, permitting entrapment of serum proteins [4]. Signals for IgM and C3 in this patient were negative, and IgG signals were more intense in the present patient than commonly seen in diabetic nephropathy. Therefore, it is unlikely that linear IgG deposition on glomerular capillary walls was caused by entrapment of serum proteins in our patient.

Because we did not observe donor-specific anti-HLA antibodies in the patient, we searched for non-HLA anti- bodies, resulting in identification of anti-MICA antibodies. MICA is expressed in endothelial cells, epithelial cells, monocytes, keratinocytes, and dendritic cells, and is not detected on the cell surface in their normal state; expression is increased by cell stress and cytokines (e.g., TNFa) [5]. The current evidence surrounding the association of MICA with transplant rejection is contradictory [6-9].

A recently developed single-antigen bead assay identified 12 autoantibodies in the patient's serum. Of these, vimentin and GSTT1 may be associated with antibodymediated rejection or graft dysfunction in solid-organ transplantation [10-13]. Vimentin is an intermediate filament protein that, when polymerized, forms the basis of the fibroblast, smooth muscle cell, and endothelial cell cytoskeletons. It participates in signal transduction, maintenance of cell structure, cell motility, and cell proliferation [14]. There is evidence showing that anti-vimentin antibodies are associated with interstitial fibrosis/ tubular atrophy and transplant glomerulopathy after kidney transplantation [10-12]. While vimentin is not expressed on the cell surface, damage to the endothelium during surgery allows it to become exposed to the immune system [10].

GSTT1 is expressed on liver and kidney cells as well as on erythrocytes. It has been reported that anti-GSTT1 antibodies could be responsible for the occurrence of antibody-mediated rejection [13] and that anti-GSTT1 antibodies are associated with de novo hepatitis after liver transplantation $[15,16]$.

As IgG signals were not detected when we incubated patient's serum with 0 -h allograft biopsy samples from donor and control, we could not confirm the deposition of autoantibodies on glomerular capillaries. However, unlike HLAs, which are constitutively expressed on the cell surface of the allograft endothelium, non-HLA autoantigens are usually cryptic. Tissue injury associated with ischemia-reperfusion and rejection creates permissive conditions for the exposure of cryptic autoantigens and allows them to bind to antigenic targets [17]. Autoantibodies may not bind to antigens on endothelial cells of 0 -h biopsy specimens if cell injury is just limited.

In conclusion, linear IgG deposition on glomerular capillaries observed in our patient may be a result of binding of non-HLA antibodies to autoantigens such as MICA, vimentin, and GSTT1, which might be permissively expressed after severe endothelial cell injury. Further studies are required to investigate and confirm the significance of non-HLA antibodies in glomerular IgG deposition. 


\section{Acknowledgement}

We would like to thank Editage (www.editage.com) for English language editing.

\section{Statement of Ethics}

This study was approved by the Ethical Review Board of our hospital (approval number: 5465).

\section{Conflict of Interest Statement}

The authors have no conflicts of interest to declare.

\section{Author Contributions}

Kenichiro Miura participated in designing the work, data collection, interpretation of data, and drafting the manuscript. Yoko Shirai participated in designing the work and collected the samples. Naoto Kaneko, Tomoo Yabuuchi, and Kiyonobu Ishizuka participated in designing the work. Shigeru Horita performed immunofluorescence studies. Miyuki Furusawa performed assays for non-HLA antibodies. Kohei Unagami, Yoichi Kakuta, and Masayoshi Okumi participated in designing the work and critical revision of the manuscript. Hideki Ishida and Kazunari Tanabe participated in critical revision of the manuscript. Junki Koike, Kazuho Honda, and Yutaka Yamaguchi participated in pathological examinations of kidney biopsy specimens and critical revision of the manuscript. Motoshi Hattori critically reviewed the manuscript and supervised the whole study process. All authors read and approved the final manuscript.

\section{References}

1 Haas M, Loupy A, Lefaucheur C, Roufosse C, Glotz D, Seron D. The Banff 2017 kidney meeting report: revised diagnostic criteria for chronic active $\mathrm{T}$ cell-mediated rejection, antibody-mediated rejection, and prospects for integrative endpoints for next-generation clinical trials. Am J Transplant. 2018 Feb; 18(2): 293-307.

2 Nickeleit V, Mengel M, Colvin RB. Renal transplant pathology. In: Jennette JC, Olson JL, Silva FG, D’Agati VD, editors. Heptinstall's pathology of the kidney. 7th ed. Philadelphia: Wolters Kluwer; 2015. p. 1321-459.

3 Sato T, Fujieda M, Tanaka E, Miyamura M, Chikamoto H, Hisano M, et al. Monitoring of Epstein-Barr virus load and antibody in pediatric renal transplant patients. Pediatr Int. 2008 Aug;50(4):454.

4 Olson JL, Laszik ZG. Diabetic nephropathy. In: Jennette JC, Olson JL, Silva FG, D’Agati VD, editors. Heptinstall's pathology of the kidney. 7th ed. Philadelphia: Wolters Kluwer; 2015. p. 897-949.

5 Lin D, Lavender H, Soilleux EJ, O'Callaghan CA. NF- $\kappa B$ regulates MICA gene transcription in endothelial cell through a genetically inhibitable control site. J Biol Chem. 2012 Feb;287(6):4299.

6 Panigrahi A, Gupta N, Siddiqui JA, Margoob A, Bhowmik D, Guleria S, et al. Post transplant development of MICA and anti-HLA antibodies is associated with acute rejection episodes and renal allograft loss. Hum Immunol. 2007 May;68(5):362-7.

7 Zou Y, Stastny P, Süsal C, Döhler B, Opelz G. Antibodies against MICA antigens and kidney-transplant rejection. N Engl J Med. 2007 Sep;357(13):1293-300.

8 Yu LX, Wang G, Fu SJ, Xiao LL, Xu J, Du CF. Anti-MICA antibodies: risk factors for sensitization and the impact on renal transplantation outcomes. Nan Fang Yi Ke Da Xue Xue Bao. 2011 Apr;31(4):615-8.

9 Solgi G, Furst D, Mytilineos J, Pourmand G, Amirzargar AA. Clinical relevance of pre and post-transplant immune markers in kidney allograft recipients: anti HLA and MICA antibodies and serum levels of sCD30 and sMICA. Transpl Immunol. 2012 Mar;26(2-3): 81-7.

10 Carter V, Shenton BK, Jaques B, Turner D, Talbot D, Gupta A, et al. Vimentin antibodies: a non-HLA antibody as a potential risk factor in renal transplantation. Transplant Proc. 2005 Mar;37(2):654.

11 Besarani D, Cerundolo L, Smith JD, Procter J, Barnardo MC, Roberts IS, et al. Role of antivimentin antibodies in renal transplantation. Transplantation. 2014 Jul;98(1):72.

12 Gunasekaran M, Maw TT, Santos RD, Shenoy S, Wellen J, Mohanakumar T. Immunoglobulin isotype switching of antibodies to vimen- tin is associated with development of transplant glomerulopathy following human renal transplantation. Transpl Immunol. 2017 Dec; 45:42.

13 Alvarez-Márquez A, Aguilera I, Gentil MA, Caro JL, Bernal G, Fernández Alonso J, et al. Donor-specific antibodies against HLA, MICA, and GSTT1 in patients with allograft rejection and $\mathrm{C} 4 \mathrm{~d}$ deposition in renal biopsies. Transplantation. 2009 Jan; 87(1):94.

14 Divanyan T, Acostaa E, Patela D, Constantinob D, Lopez-Soler RI. Anti-vimentin antibodies in transplant and disease. Hum Immunol. 2019 Sep;80(9):602-7.

15 Aguilera I, Sousa JM, Gavilán F, Bernardos A, Wichmann I, Nuñez-Roldán A. Glutathione S-transferase T1 mismatch constitutes a risk factor for de novo immune hepatitis after liver transplantation. Liver Transpl. 2004 Sep; 10(9):1166.

16 Aguilera I, Martinez-Bravo MJ, Sousa JM, Pozo-Borrego AJ, Núñez-Roldán A. IgG subclass profile among anti-glutathione S-transferase $\mathrm{T} 1$ antibodies in post-transplant de novo immune hepatitis. Clin Transplant. 2016 Mar;30(3):210.

17 Cardinal H, Dieudé M, Hébert M-J. The emerging importance of non-HLA autoantibodies in kidney transplant complications. J Am Soc Nephrol. 2017 Feb;28(2):400. 